\title{
Critical Pathway Improves Arrival-In-Cath-Lab Interval for Patients With Acute Myocardial Infarction in the Emergency Department
}

\author{
Iwao Nakamura, MD; Shingo Hori, MD; Masaru Suzuki, MD; \\ Yasushi Asakura, MD*; Tsutomu Yoshikawa, MD*; \\ Satoshi Ogawa, MD*; Naoki Aikawa, MD
}

\begin{abstract}
A newly developed critical pathway has been applied to emergency medical care of patients with acute myocardial infarction (AMI) in the emergency department. The arrival-in-cath-lab interval (ACI) was selected as a clinical indicator of quality assurance, according to American Heart Association and American College of Cardiology guidelines. This report describes the first experience of applying the critical pathway to patients with AMI in an emergency department in Japan. The ACI of 35 AMI patients who underwent primary percutaneous transluminal coronary angioplasty during a 24-month period following application of the pathway were compared with those of 50 AMI patients during the 48 months before application of the pathway. The median ACI value was significantly reduced from $65.5 \mathrm{~min}$ to $50.0 \mathrm{~min}$. Also, the proportion of patients with acceptably short ACI $(<60 \mathrm{~min})$ significantly improved from $42.0 \%$ to $68.6 \%$. (Jpn Circ J 2001; 65: 849-852)
\end{abstract}

Key Words: Acute myocardial infarction; Arrival-in-cath-lab interval; Clinical indicators; Critical pathway; Emergency department

C ritical pathways are management plans that display goals for patients and provide the sequence of optimal efficiency! They have been developed not only for nursing, but also for medical care, to reduce costs and improve quality of care. Many pathways for cardiovascular diseases have been developed to date, including those for emergency cardiovascular care?

In the treatment of acute myocardial infarction (AMI), early recanalization of the infarct-related coronary artery is the first priority and there is considerable evidence of the significance of reducing the time interval between symptom onset and initiation of thrombolytic therapy (onset-to-drug time $)^{3-6}$ or balloon inflation in percutaneous transluminal coronary angioplasty (PTCA) (onset-to-balloon time)? Both the American Heart Association (AHA) and the American College of Cardiology (ACC) recommend in their 1996 guidelines $^{8}$ that the goal of emergency care for AMI is a door-to-drug (thrombolytic agent) interval less than $30 \mathrm{~min}$ or an arrival-in-cath-lab interval (ACI) of less than $60 \mathrm{~min}$. Cannon et al reported that after implementation of the AMI protocol in the Emergency Department of Brigham and Women's Hospital? the door-to-drug time was significantly reduced from $76 \mathrm{~min}$ to $29 \mathrm{~min}$.

However, in Japan, measurement of time intervals as clinical indicators and application of critical pathways are not yet popular among physicians. The purpose of this study was to clarify whether application of a critical pathway in the emergency department (ED) of an urban

(Received April 11, 2001; revised manuscript received June 7, 2001; accepted June 26, 2001)

Emergency Medicine and *Cardiology Division, Department of Internal Medicine, Keio University, Tokyo, Japan

Mailing address: Shingo Hori, MD, Emergency Medicine, Keio University, School of Medicine, 35 Shinanomachi, Shinjuku-ku, Tokyo 160-8582, Japan. E-mail: shingo@sc.itc.keio.ac.jp university hospital would reduce the ACI in the management of patients with AMI.

\section{Methods}

The study was conducted prospectively with ACI as the outcome measured among patients with AMI who presented in 1999 and 2000. The data were compared to those obtained from historical control patients seen between 1995 and 1998. Between the 2 periods, the systems of emergency medical services were similar; all patients who presented to the ED by ambulance were initially treated by emergency attending physicians who supervised emergency residents.

\section{The Critical Pathway (Table 1)}

The critical pathway was used in the ED as of January 1999. It is actually a sheet of paper in the emergency room that describes each step of the initial evaluation and treatment according to the time course, from the patient's arrival in the ED until arrival in the catheterization laboratory (cath lab). The emergency physicians and residents who use this pathway have to write the actual time taken for each step of medical care carried out in the ED. After the patient arrives in the cath lab, the physician calculates the ACI and fills out the sheet, which is then filed in the hospital chart. Additionally, the physician must describe the actual reason for prolongation of the time interval if the $\mathrm{ACI}$ is more than $60 \mathrm{~min}$.

\section{Enrolled Patients}

From January 1995 to December 2000, 19,279 patients were brought to the Department of Emergency Medicine at Keio University Hospital by ambulance. Among them, 102 patients were diagnosed as having AMI with possible indications for reperfusion therapy: (1) chest pain persisting 
Table 1 Critical Pathway for AMI Patients in the Emergency Department

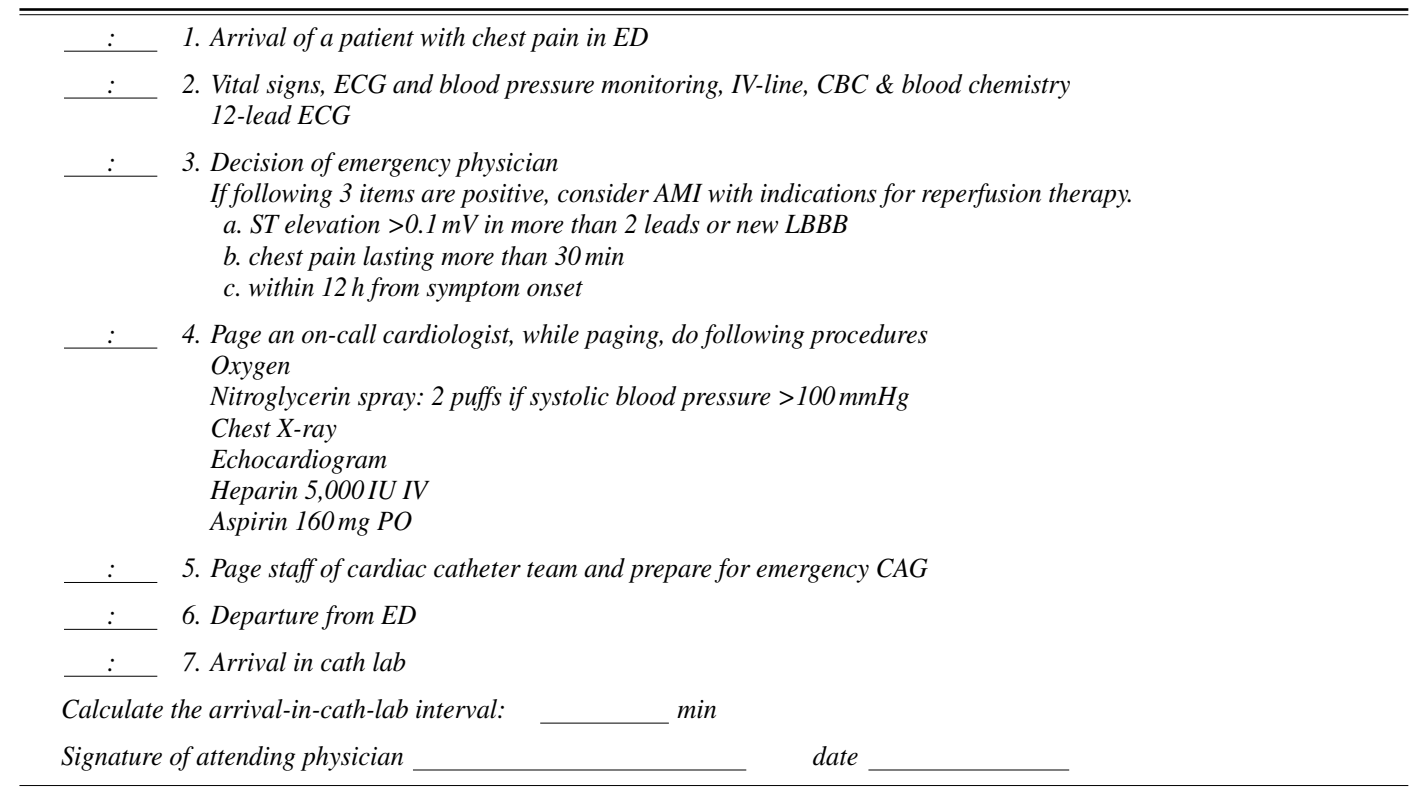

Table 2 Patient Characteristics

\begin{tabular}{lccc}
\hline \hline & $\begin{array}{c}\text { Pre-pathway group } \\
(1995-1998, n=50)\end{array}$ & $\begin{array}{c}\text { Post-pathway group } \\
(1999-2000, n=35)\end{array}$ & p value \\
\hline Age (years)* & $63.0(10-90)$ & $64.0(40-91)$ & 0.696 \\
Male & $40(80.0 \%)$ & $29(82.9 \%)$ & 0.740 \\
Onset-to-hospital arrival interval (min)* & $118.0(22-643)$ & $116.0(17-450)$ & 0.517 \\
Infarct-related coronary artery & & & \\
LMT & $2(4.0 \%)$ & $2(5.7 \%)$ & 0.653 \\
RCA & $24(48.0 \%)$ & $18(51.4 \%)$ & 0.389 \\
LAD & $21(42.0 \%)$ & $13(37.1 \%)$ & \\
LCX & $3(6.0 \%)$ & $0(0 \%)$ & 0.712 \\
Normal CAG & $0(0 \%)$ & $2(5.7 \%)$ & 0.712 \\
Primary PTCA & $46(92.0 \%)$ & $31(88.6 \%)$ & \\
Final diagnosis & $46(92.0 \%)$ & $31(88.6 \%)$ & \\
AMI & $3(6.0 \%)$ & $1(2.9 \%)$ & \\
Unstable angina & $1(2.0 \%)$ & $1(2.9 \%)$ & \\
Vasospastic angina & $0(0 \%)$ & $1(2.9 \%)$ & \\
Myocarditis & $0(0 \%)$ & $1(2.9 \%)$ & \\
Aortic dissection & $(0 \%)$ & \\
\hline
\end{tabular}

*Values for age and onset-to-hospital arrival interval are expressed as the median, with range in parentheses.

Table 3 Arrival-in-Cath-Lab Intervals (ACI)

\begin{tabular}{lccc}
\hline \hline & $\begin{array}{c}\text { Pre-pathway group } \\
(n=50)\end{array}$ & $\begin{array}{c}\text { Post-pathway group } \\
(n=35)\end{array}$ & p value \\
\hline$A C I(\min )^{*}$ & $65.5(26-140)$ & $50.0(15-116)$ & 0.0331 \\
$A C I<60$ min & $21(42.0 \%)$ & $24(68.6 \%)$ & 0.0157 \\
\hline
\end{tabular}

*Values for ACI are expressed as the median and range (in parentheses).

more than $30 \mathrm{~min}$ with onset within $12 \mathrm{~h}$; and (2) ST segment elevation $(\geq 0.1 \mathrm{mV})$ in 2 or more contiguous ECG leads. Eighty-five patients were directly transported to the cath lab, underwent emergency coronary angiography (CAG) and were enrolled in this study. In the remaining 17 patients, emergency CAG was not performed because of (1) lack of availability of hospital beds or transportation to other hospitals (3 patients), (2) failure to obtain informed consent (2 patients), (3) institution of intravenous thrombolysis ( 2 patients) and (4) unknown reasons (10 patients).
The 85 patients who underwent emergency CAG were divided into 2 groups: those treated in the absence of the critical pathway (pre-pathway group, 1995-1998, $n=50$ ), and those treated under the guidance of the critical pathway (post-pathway group, 1999-2000, $\mathrm{n}=35$ ).

\section{Statistical Analysis}

Patient age, onset-to-hospital arrival interval and ACI were compared between the pre-pathway and post-pathway groups by Mann-Whitney's U test. Gender, infarct-related 
arteries and the incidences of primary PTCA and an acceptable ACI (within $60 \mathrm{~min}$ ) were compared between the 2 groups by chi-square analyses.

\section{Results}

Age and gender, onset to hospital arrival interval, infarct-related arteries, incidence of primary PTCA, and the incidence of a final diagnosis of AMI were comparable between the 2 groups (Table 2).

Following institution of the critical pathway, the median ACI was significantly shortened from $65.5 \mathrm{~min}$ (26-140 $\mathrm{min}$ ) in the pre-pathway group to $50.0 \mathrm{~min}(15-116 \mathrm{~min})$ in the post-pathway group (Table 3), which fulfilled the goal of the AHA/ACC Guidelines (ACI $<60 \mathrm{~min}$ ) of a significant improvement (from $42.0 \%$ to $68.6 \%$ ).

In the post-pathway group, the ACI of 11 patients was more than $60 \mathrm{~min}$ and 5 patients had an ACI of more than $90 \mathrm{~min}$. The reasons for the prolonged ACI in the 5 patients were delay in diagnosis in 2 patients, delay in the response of the cardiac catheter team in 2 patients and unavailability of the cath lab in 1 patient.

\section{Discussion}

Application of a critical pathway in the emergency treatment of patients suspected of having AMI has been shown to significantly decrease the ACI and increase the number of patients meeting the ACC/AHA criterion of an ACI less than $60 \mathrm{~min}$. Reports from the United States ${ }^{9}$ and the United Kingdom ${ }^{10}$ indicate that application of critical pathways or education of emergency staff reduces the door-to-drug (or door-to-needle) time for AMI patients receiving intravenous thrombolytic therapy, and because intravenous thrombolysis usually involves a range of procedures performed by emergency physicians, door-to-drug time has been used as an indicator of the quality of medical care in the ED. The critical pathway has been shown to be necessary for continuous quality improvement of ED care in Western countries, but in Japan, where the incidence of primary PTCA is equal to or more than that of intravenous thrombolytic therapy, there have been few systematic efforts as yet to reduce the interval to reperfusion in the ED. This is the first report from Japan on the application of a critical pathway in the ED to reduce the ACI in the setting of primary PTCA as the emergency treatment goal.

AMI is one of the common diseases in emergency medicine and so it is necessary to establish realistic treatment guidelines in every institute. Critical pathways clarify the goal of therapy not only for emergency physicians or cardiologists, but for all medical personnel involved in the emergency patient's care. It also promotes good collaboration between medical personnel.

Although our data demonstrated that application of a critical pathway in the ED is as effective in Japan as in Western countries, it was difficult to clarify why the pathway was effective in reducing the ACI because medical charts before the introduction of the pathway rarely clarified which treatment process was the true cause of the delay. 'Physician's delay' (patient's arrival-to-physician evaluation interval) is considered to be one of the reasons for in-hospital delay! ${ }^{12,13}$ In 32 patients (64\%) of prepathway group, the actual time of ECG diagnosis was recorded in the medical charts and therefore could be analyzed. The median time interval between patient arrival and ECG diagnosis was $6.5 \mathrm{~min}$, which was not significantly different from the interval of $5.0 \mathrm{~min}$ for the postpathway group. therefore, we speculated that the cause of the prolonged ACI in the pre-pathway group may in the complicated clinical process following the recording of the ECG in the ED. This is most likely where the pathway improves the ACI because it clearly states the process of patient care, standardizes the care and rationalizes the sequence of orders of treatment by medical personnel. Before the implementation of the critical pathway, some emergency physicians performed echocardiography to confirm their diagnosis before deciding to page cardiologists and then the on-call cardiologists repeated the test to confirm the diagnosis for themselves. After application of the critical pathway, echocardiography was standardized so as to consistently be done only after paging the on-call cardiologist (Table 1).

Recently, a new guideline for acute coronary syndrome was published in the International Guidelines 2000 for Cardiopulmonary Resuscitation and Emergency Cardiovascular Care!1 The ACI was changed to door-to-balloon inflation time $(90 \pm 30 \mathrm{~min})$ as the indicator of patient treatment with primary PTCA. Therefore, the indicator of patient treatment with the critical pathway will in the future be changed to door-to-balloon time.

\section{Study Limitations}

In the present study, the long-term prognosis of patients was not included in the analysis. The peak serum creatine kinase $(2,942 \pm 2,228$ vs $3,213 \pm 3,065 \mathrm{IU} / \mathrm{L})$ and in-hospital mortality (10.0 vs $5.7 \%$ ) did not differ significantly between the pre-pathway and post-pathway groups. Kelion et al also reported that reduction of the door-to-drug time did not improve the 1-month mortality rate of 142 AMI patients. ${ }^{10}$ However, the ACI is a component of the onset-to-reperfusion interval, which has been documented to be a clinical indicator associated with lower mortality?-6 Cannon et al also proved that reducing the door-to-balloon time improved in-hospital mortality in a large scale (27,000 patients) study? These data support the selection of ACI as the clinical indicator of emergency care because monitoring of the ACI under the guidance of the critical pathway will improve the quality of emergency medical care not only from the viewpoint of time reduction, but also by standardizing the emergency care of patients with AMI. A large scale, randomized study is needed to confirm that the critical pathway reduces the mortality of patients with AMI in Japan.

\section{References}

1. Pearson SD, Goulart-Fisher D, Lee TH: Critical pathways as a strategy for improving care: Problems and potential. Ann Intern Med 1995; 123: $941-948$

2. Every NR, Hochman J, Becker R, Kopecky S, Cannon CP: Critical pathways: A review. Committee on Acute Cardiac Care, Council on Clinical Cardiology, American Heart Association. Circulation 2000; 101: $461-465$

3. Gruppo Italiano per lo Studio della Streptochinasi nell'Infarto Miocardico (GISSI): Effectiveness of intravenous thrombolytic treatment in acute myocardial infarction. Lancet 1986; 1: 397-402

4. Fibrinolytic Therapy Trialists' (FTT) Collaborative Group: Indications for fibrinolytic therapy in suspected acute myocardial infarction: Collaborative overview of early mortality and major morbidity results from all randomized trials of more than 1000 patients. Lancet 1994; 343: $311-322$

5. The GUSTO Angiographic Investigators: The effects of tissue plasminogen activator, streptokinase, or both on coronary-artery patency, ventricular function and survival after acute myocardial infarction. $N$ Engl J Med 1993; 329: 1615-1622 
6. Goldberg RJ, Mooradd M, Gurwitz JH, Rogers WJ, French WJ, Barron HV, et al: Impact of time to treatment with tissue plasminogen activator on morbidity and mortality following acute myocardial infarction (The second National Registry of Myocardial Infarction). Am J Cardiol 1998; 82: 259-264

7. Cannon CP, Gibson CM, Lambrew CT, Shoulz DA, Levy D, French WJ, et al: Relationship of symptom-onset-to-balloon time and doorto-balloon time with mortality in patients undergoing angioplasty for acute myocardial infarction. JAMA 2000; 283: 2941-2947

8. Ryan TJ, Anderson JL, Antman EM, Braniff BA, Brooks NH, Califf RM, et al: ACC/AHA guidelines for the management of patients with acute myocardial infarction: A report of the American College of Cardiology/American Heart Association Task Force on Practice Guidelines (Committee on Management of Acute Myocardial Infarction). J Am Coll Cardiol 1996; 28: 1328-1428

9. Cannon CP, Antman EM, Walls R, Braunwald E: Time as an adjunctive agent to thrombolytic therapy. J Thromb Thrombolysis 1994; 1:
27-34

10. Kelion AD, Banning AP, Shahi M, Bell JA: The effect of reduction of door-to-needle times on the administration of thrombolytic therapy for acute myocardial infarction. Postgrad Med J 1998; 74: $533-536$

11. Guidelines 2000 for Cardiopulmonary Resuscitation and Emergency Cardiovascular Care. Part 7: The Era of Reperfusion: section 1: Acute coronary syndromes (acute myocardial infarction). The American Heart Association in collaboration with the International Liaison Committee on Resuscitation. Circulation 2000; 102(Suppl 1): I-172I-203

12. Krall SP, Reese IV CL, Donahue L: Effect of continuous quality improvement methods on reducing triage to thrombolytic interval for acute myocardial infarction. Acad Emerg Med 1995; 2: 603-609

13. Bracken J: Reducing door-to-needle time: Treatment delay versus presentation delay. Clin Cardiol 1997; 20(Suppl III): III-21-III-25 